\title{
Computing center conditions for non-analytic ninth degree vector fields with constant angular speed
}

Qingjun Ren, Yikun Huang and Feng Li*

\section{*Correspondence:}

092101013@csu.edu.cn

School of Science, Linyi University,

Linyi, Shandong 276005, P.R. China

\begin{abstract}
We investigate the planar ninth degree non-analytic Poincare systems which have center-focus equilibriums at the origins. Those systems could be changed into analytic systems by two transformations with the help of computer algebra system MATHEMATICA. The conditions of uniform isochronous center are obtained. Furthermore, we prove that five limit cycles could be bifurcated from the origin. MSC: $34 \mathrm{C05} ; 34 \mathrm{CO}$
\end{abstract}

Keywords: non-analytic; center-focus problem; Lyapunov constant; uniform isochronous center

\section{Introduction}

One of open problems for planar polynomial differential systems is how to characterize their centers and isochronous centers. Article [1] points out that 'A center of an analytic system is isochronous if and only if there exists an analytic change of coordinates such that the original system is reduced to a linear system, so an isochronous center is also called a linearizable center. If all solutions of the system rotate around the origin, and their angular speeds are constant along rays, finding isochronous centers is equivalent to proving the existence of centers, and the center is called to be a uniform isochronous center. Polynomial systems with a uniform isochronous center can always be written in the following form:

$$
\begin{aligned}
& \frac{d x}{d t}=-y+x H(x, y), \\
& \frac{d y}{d t}=x+y H(x, y),
\end{aligned}
$$

where $H(x, y)$ is a polynomial.

The system has a unique fixed point $O(0,0)$ which is a center for the linear approximation and enjoys the property of uniform isochronicity: the motion along the trajectories occurs with the constant angular velocity equal to one. The fixed point can then be a focus or a center. If it is a center, it will automatically be isochronous. The problem of characterizing the centers of polynomial uniformly isochronous systems attracted the attention of many authors. When $H(x, y)$ is a homogeneous polynomial, the problem of characterizing

\section{囪 Springer}

(c) 2012 Ren et al.; licensee Springer. This is an Open Access article distributed under the terms of the Creative Commons Attribution License (http://creativecommons.org/licenses/by/2.0), which permits unrestricted use, distribution, and reproduction in any medium, provided the original work is properly cited. 
the centers has been solved; see [2]. But when $H(x, y)$ is a nonhomogeneous polynomial, the problem remains open; see [3-6] and the references therein.

In this work, we study the uniformly isochronous system, not necessarily polynomial one:

$$
\begin{aligned}
\frac{d x}{d t}= & -y+x\left(\left(x^{2}+y^{2}\right)^{\lambda-1}\left(A_{20} x^{2}+A_{11} x y+A_{02} y^{2}\right)+\left(x^{2}+y^{2}\right)^{4(\lambda-1)}\left(A_{80} x^{8}+A_{71} x^{7} y\right.\right. \\
& \left.\left.+A_{62} x^{6} y^{2}+A_{53} x^{5} y^{3}+A_{44} x^{4} y^{4}+A_{35} x^{3} y^{5}+A_{26} x^{2} y^{6}+A_{17} x y^{7}+A_{08} y^{8}\right)\right), \\
\frac{d y}{d t}= & x+y\left(\left(x^{2}+y^{2}\right)^{\lambda-1}\left(A_{20} x^{2}+A_{11} x y+A_{02} y^{2}\right)+\left(x^{2}+y^{2}\right)^{4(\lambda-1)}\left(A_{80} x^{8}+A_{71} x^{7} y\right.\right. \\
& \left.\left.+A_{62} x^{6} y^{2}+A_{53} x^{5} y^{3}+A_{44} x^{4} y^{4}+A_{35} x^{3} y^{5}+A_{26} x^{2} y^{6}+A_{17} x y^{7}+A_{08} y^{8}\right)\right) .
\end{aligned}
$$

Similar systems have been investigated; see [7-12]. The main goal of this paper is to use the integral factor method theory to distinguish center-focus and give the conditions of a uniform isochronous center.

As far as we know, there were few results about a nonanalytic Poincarè system. This paper is divided into four sections. In Section 2, we restate some known results necessary to demonstrate the main results. In Section 3, we use the recursive algorithm to obtain the conditions of a uniform isochronous center for the system. In Section 4, the bifurcations of limit circles are investigated, and five limit circles could be bifurcated from the origin.

\section{Preliminary knowledge}

The ideas of this section come from [13], where the center-focus problem of critical points in the planar dynamical systems are studied. We first recall the related notions and results. For more details, please refer to [13].

In [13], the authors defined a complex center and a complex isochronous center for the following complex system:

$$
\begin{aligned}
& \frac{d z}{d T}=z+\sum_{k=2}^{\infty} \sum_{\alpha+\beta=k} a_{\alpha \beta} z^{\alpha} w^{\beta}=Z(z, w), \\
& \frac{d w}{d T}=-w-\sum_{k=2}^{\infty} \sum_{\alpha+\beta=k} b_{\alpha \beta} w^{\alpha} z^{\beta}=-W(z, w),
\end{aligned}
$$

and gave two recursive algorithms to determine necessary conditions for a center and an isochronous center. We now restate the definitions and algorithms.

Lemma 2.1 [13] For system (2.1), we can derive uniquely the following formal series:

$$
\xi=z+\sum_{k+j=2}^{\infty} c_{k j} z^{k} w^{j}, \quad \eta=w+\sum_{k+j=2}^{\infty} d_{k j} w^{k} z^{j}
$$

where $c_{k+1, k}=d_{k+1, k}=0, k=1,2, \ldots$, such that

$$
\frac{d \xi}{d T}=\xi+\sum_{j=1}^{\infty} p_{j} \xi^{j+1} \eta^{j}, \quad \frac{d \eta}{d T}=-\eta-\sum_{j=1}^{\infty} q_{j} \eta^{j+1} \xi^{j} .
$$


Definition 2.1 [13] Let $\mu_{0}=0, \mu_{k}=p_{k}-q_{k}, \tau_{k}=p_{k}+q_{k}, k=1,2, \ldots \mu_{k}$ is called the $k$ th singular point quantity of the origin of system (2.1) and $\tau_{k}$ is called the $k$ th period constant of the origin of system (2.1).

Theorem 2.1 [13] For system (2.1), the origin is a complex center if and only if $\mu_{k}=0$, $k=1,2, \ldots$. The origin is a complex isochronous center if and only if $\mu_{k}=\tau_{k}=0, k=1,2, \ldots$.

Theorem 2.2 [13] For system (2.1), we can derive successively the terms of the following formal series:

$$
M(z, w)=\sum_{\alpha+\beta=0}^{\infty} c_{\alpha \beta} z^{\alpha} w^{\beta}
$$

such that

$$
\frac{\partial(M Z)}{\partial z}-\frac{\partial(M W)}{\partial w}=\sum_{m=1}^{\infty}(m+1) \mu_{m}(z w)^{m}
$$

where $c_{00}=1, \forall c_{k k} \in R, k=1,2, \ldots$, and for any integer $m, \mu_{m}$ is determined by the following recursive formulae:

$$
\begin{aligned}
& c_{\alpha \beta}=\frac{1}{\beta-\alpha} \sum_{k+j=3}^{\alpha+\beta+2}\left[(\alpha+1) a_{k, j-1}-(\beta+1) b_{j, k-1}\right] c_{\alpha-k+1, \beta-j+1}, \\
& \mu_{m}=\sum_{k+j=3}^{2 m+2}\left(a_{k, j-1}-b_{j, k-1}\right) c_{m-k+1, m-j+1} .
\end{aligned}
$$

\section{Conditions of a uniform isochronous center}

Now, the conditions of a uniform isochronous center of a class of systems will be investigated with the help of Mathematics.

Through the transformations

$$
\begin{aligned}
& u=x\left(x^{2}+y^{2}\right)^{\frac{\lambda-1}{2}}, \quad v=y\left(x^{2}+y^{2}\right)^{\frac{\lambda-1}{2}}, \\
& z=u+i v, \quad w=u-i v, \quad T=i t, \quad i=\sqrt{-1},
\end{aligned}
$$

system (1.2) can be transformed into the following system:

$$
\begin{aligned}
\frac{d z}{d T}= & z-\frac{1}{4} A_{11} \lambda z^{3}+\frac{1}{4} A_{11} \lambda z w^{2} \\
& +\frac{1}{256} i\left(-A_{08}-i A_{17}+A_{26}+i A_{35}-A_{44}-i A_{53}+A_{62}+i A_{71}-A_{80}\right) \lambda z^{9} \\
& +\frac{1}{1,290,240} i\left(40,320 A_{08}+30,240 i A_{17}-20,160 A_{26}-10,080 i A_{35}-10,080 i A_{53}\right. \\
& \left.+20,160 A_{62}+30,240 i A_{71}-40,320 A_{80}\right) \lambda z^{8} w \\
& +\frac{1}{368,640} i\left(-40,320 A_{08}-20,160 i A_{17}+5,760 A_{26}-2,880 i A_{35}+5,760 A_{44}\right.
\end{aligned}
$$




$$
\begin{aligned}
& \left.+2,880 i A_{53}+5,760 A_{62}+20,160 i A_{71}-40,320 A_{80}\right) \lambda z^{7} w^{2} \\
& +\frac{1}{184,320} i\left(40,320 A_{08}+10,080 i A_{17}+2,880 A_{26}+4,320 i A_{35}+4,320 i A_{53}\right. \\
& \left.-2,880 A_{62}+10,080 i A_{71}-40,320 A_{80}\right) \lambda z^{6} w^{3} \\
& +\frac{1}{147,456} i\left(-40,320 A_{08}-5,760 A_{26}-3,456 A_{44}\right. \\
& \left.-5,760 A_{62}-40,320 A_{80}\right) \lambda z^{5} w^{4} \\
& +\frac{1}{184,320} i\left(40,320 A_{08}-10,080 i A_{17}+2,880 A_{26}-4,320 i A_{35}-4,320 i A_{53}\right. \\
& \left.-2,880 A_{62}-10,080 i A_{71}-40,320 A_{80}\right) \lambda z^{4} w^{5} \\
& +\frac{1}{368,640} i\left(-40,320 A_{08}+20,160 i A_{17}+5,760 A_{26}+2,880 i A_{35}+5,760 A_{44}\right. \\
& \left.-2,880 i A_{53}+5,760 A_{62}-20,160 i A_{71}-40,320 A_{80}\right) \lambda z^{3} w^{6} \\
& +\frac{1}{1,290,240} i\left(40,320 A_{08}-30,240 i A_{17}-20,160 A_{26}+10,080 i A_{35}+10,080 i A_{53}\right. \\
& \left.+20,160 A_{62}-30,240 i A_{71}-40,320 A_{80}\right) \lambda z^{2} w^{7} \\
& +\frac{1}{10,321,920} i\left(-40,320 A_{08}+40,320 i A_{17}+40,320 A_{26}-40,320 i A_{35}\right. \\
& \left.-40,320 A_{44}+40,320 i A_{53}+40,320 A_{62}-40,320 i A_{71}-40,320 A_{80}\right) \lambda z w^{8} ; \quad \text { (3.1) } \\
& \frac{d w}{d T}=-w+\frac{1}{256} i\left(64 A_{02}-64 i A_{11}-64 A_{20}\right) \lambda w^{3}+\frac{1}{256} i\left(-128 A_{02}-128 A_{20}\right) \lambda w^{2} z \\
& +\frac{1}{512} i\left(128 A_{02}+128 i A_{11}-128 A_{20}\right) \lambda w z^{2} \\
& +\frac{1}{256} i\left(-A_{08}+i A_{17}+A_{26}-i A_{35}-A_{44}+i A_{53}+A_{62}-i A_{71}-A_{80}\right) \lambda w^{9} \\
& +\frac{1}{1,290,240} i\left(40,320 A_{08}-30,240 i A_{17}-20,160 A_{26}+10,080 i A_{35}+10,080 i A_{53}\right. \\
& \left.+20,160 A_{62}-30,240 i A_{71}-40,320 A_{80}\right) \lambda w^{8} z \\
& +\frac{1}{368,640} i\left(-40,320 A_{08}+20,160 i A_{17}+5,760 A_{26}+2,880 i A_{35}+5,760 A_{44}\right. \\
& \left.-2,880 i A_{53}+5,760 A_{62}-20,160 i A_{71}-40,320 A_{80}\right) \lambda w^{7} z^{2} \\
& +\frac{1}{184,320} i\left(40,320 A_{08}-10,080 i A_{17}+2,880 A_{26}-4,320 i A_{35}-4,320 i A_{53}\right. \\
& \left.-2,880 A_{62}-10,080 i A_{71}-40,320 A_{80}\right) \lambda w^{6} z^{3} \\
& +\frac{1}{147,456} i\left(-40,320 A_{08}-5,760 A_{26}-3,456 A_{44}\right. \\
& \left.-5,760 A_{62}-40,320 A_{80}\right) \lambda w^{5} z^{4} \\
& +\frac{1}{184,320} i\left(40,320 A_{08}+10,080 i A_{17}+2,880 A_{26}+4,320 i A_{35}+4,320 i A_{53}\right. \\
& \left.-2,880 A_{62}+10,080 i A_{71}-40,320 A_{80}\right) \lambda w^{4} z^{5}
\end{aligned}
$$




$$
\begin{aligned}
& +\frac{1}{368,640} i\left(-40,320 A_{08}-20,160 i A_{17}+5,760 A_{26}-2,880 i A_{35}+5,760 A_{44}\right. \\
& \left.+2,880 i A_{53}+5,760 A_{62}+20,160 i A_{71}-40,320 A_{80}\right) \lambda w^{3} z^{6} \\
& +\frac{1}{1,290,240} i\left(40,320 A_{08}+30,240 i A_{17}-20,160 A_{26}-10,080 i A_{35}-10,080 i A_{53}\right. \\
& \left.+20,160 A_{62}+30,240 i A_{71}-40,320 A_{80}\right) \lambda w^{2} z^{7} \\
& +\frac{1}{10,321,920} i\left(-40,320 A_{08}+40,320 i A_{17}+40,320 A_{26}+40,320 i A_{35}\right. \\
& \left.-40,320 A_{44}-40,320 i A_{53}+40,320 A_{62}+40,320 i A_{71}-40,320 A_{80}\right) \lambda w z^{8} .
\end{aligned}
$$

First, we consider a special case of system (1.2),

$$
\begin{aligned}
\frac{d x}{d t}= & -y+x\left(\left(x^{2}+y^{2}\right)^{\lambda-1} A_{11} x y+\left(x^{2}+y^{2}\right)^{4(\lambda-1)}\left(A_{80} x^{8}+A_{71} x^{7} y\right.\right. \\
& \left.\left.+A_{62} x^{6} y^{2}+A_{53} x^{5} y^{3}+A_{44} x^{4} y^{4}+A_{35} x^{3} y^{5}+A_{26} x^{2} y^{6}+A_{17} x y^{7}+A_{08} y^{8}\right)\right), \\
\frac{d y}{d t}= & x+y\left(\left(x^{2}+y^{2}\right)^{\lambda-1} A_{11} x y+\left(x^{2}+y^{2}\right)^{4(\lambda-1)}\left(A_{80} x^{8}+A_{71} x^{7} y\right.\right. \\
& \left.\left.+A_{62} x^{6} y^{2}+A_{53} x^{5} y^{3}+A_{44} x^{4} y^{4}+A_{35} x^{3} y^{5}+A_{26} x^{2} y^{6}+A_{17} x y^{7}+A_{08} y^{8}\right)\right) .
\end{aligned}
$$

Namely, we discuss the calculation of singular point quantities and center conditions at the origin of system (3.1) when $A_{20}=A_{02}=0$. Applying the recursive formulae in Theorem 2.2, we compute singular point quantities and simplify them, then we have the following.

Theorem 3.1 The first eight singular point quantities at the origin of system (3.1) $\left.\right|_{A_{20}=A_{02}=0}$ are as follows:

$$
\begin{aligned}
& u_{1}=0, \\
& u_{2}=0, \\
& u_{3}=0 \\
& u_{4}=-\frac{i}{64}\left(35 A_{08}+5 A_{26}+3 A_{44}+5 A_{62}+35 A_{80}\right) \lambda, \\
& u_{5}=-\frac{3 i}{64} A_{11}\left(14 A_{08}+A_{26}-A_{62}-14 A_{80}\right) \lambda^{2}, \\
& u_{6}=-\frac{i}{32} A_{11}^{2}\left(7 A_{08}+A_{62}+21 A_{80}\right) \lambda^{3}, \\
& u_{7}=-\frac{5 i}{32} A_{11}^{3}\left(A_{08}-A_{80}\right) \lambda^{4}, \\
& u_{8}=-\frac{15 i}{128} A_{11}^{4} A_{80} \lambda^{5} .
\end{aligned}
$$

From Theorem 3.1, we get the following. 
Theorem 3.2 For system (3.1) $\left.\right|_{A_{20}=A_{02}=0}$, the first eight singular point quantities are zero if and only if one of the following conditions holds:

$$
\begin{aligned}
& A_{11}=0, \quad 35 A_{08}+5 A_{26}+3 A_{44}+5 A_{62}+35 A_{80}=0 ; \\
& A_{08}=A_{26}=A_{44}=A_{62}=A_{80}=0 .
\end{aligned}
$$

We can readily verify the following.

Theorem 3.3 For system (3.1) $\left.\right|_{A_{20}=A_{02}=0}$, all the singular point quantities at the origin are zero if and only if the first eight singular point quantities are zero, i.e., one of the conditions in Theorem 3.2 holds. Correspondingly, the conditions in Theorem 3.2 are the center conditions of the origin.

Proof If condition (3.4) holds, system (3.1) $\left.\right|_{A_{20}=A_{02}=0}$ has an analytic first integral

$$
\begin{aligned}
f= & \frac{1}{3,072 z^{5} w^{5}}\left(768+3\left(A_{17}-A_{35}+A_{53}-A_{71}-128 i A_{80}\right) \lambda z^{8}\right. \\
& -8\left(3 A_{17}-A_{35}-A_{53}+3 A_{71}\right) \lambda z^{7} w+12\left(7 A_{17}+A_{35}-A_{53}-7 A_{71}\right) \lambda z^{6} w^{2} \\
& -24\left(7 A_{17}+3 A_{35}+3 A_{53}+7 A_{71}\right) \lambda z^{5} w^{3}-24\left(7 A_{17}+3 A_{35}+3 A_{53}+7 A_{71}\right) \lambda z^{3} w^{5} \\
& +12\left(7 A_{17}+A_{35}-A_{53}-7 A_{71}\right) \lambda z^{2} w^{6}-8\left(3 A_{17}-A_{35}-A_{53}+3 A_{71}\right) \lambda z w^{7} \\
& \left.+3\left(A_{17}-A_{35}+A_{53}-A_{71}+128 i A_{80}\right) \lambda w^{8}\right) .
\end{aligned}
$$

In the case (3.5), system (3.1) $\left.\right|_{A_{20}=A_{02}=0}$ could be written as

$$
\begin{aligned}
\frac{d x}{d t}= & -y+x\left(\left(x^{2}+y^{2}\right)^{\lambda-1} A_{11} x y+\left(x^{2}+y^{2}\right)^{4(\lambda-1)}\right. \\
& \left.\times\left(A_{71} x^{7} y+A_{53} x^{5} y^{3}+A_{35} x^{3} y^{5}+A_{17} x y^{7}\right)\right), \\
\frac{d y}{d t}= & x+y\left(\left(x^{2}+y^{2}\right)^{\lambda-1} A_{11} x y+\left(x^{2}+y^{2}\right)^{4(\lambda-1)}\right. \\
& \left.\times\left(A_{71} x^{7} y+A_{53} x^{5} y^{3}+A_{35} x^{3} y^{5}+A_{17} x y^{7}\right)\right) .
\end{aligned}
$$

It is reversible and its trajectories are symmetric with respect to both coordinate axes.

Theorem 3.4 The origin of system (3.1) $\left.\right|_{A_{20}=A_{02}=0}$ is a uniform isochronous center if and only if one of the conditions in Theorem 3.3 holds.

In the previous discussion, we considered a particular case. Now, we consider the general system (1.2). Applying the recursive formulae in Theorem 2.2, we compute singular point quantities and simplify them. The first one singular point quantities at the origin of system (3.1) is

$$
u_{1}=-i\left(A_{20}+A_{02}\right) \lambda .
$$


If $A_{20}=-A_{02}$, then the change of variables $\tilde{x}=x \cos \theta+y \sin \theta, \tilde{y}=-x \sin \theta+y \cos \theta$ with $\theta$ defined from the condition

$$
A_{20} \cos ^{2} \theta+A_{11} \sin \theta \cos \theta+A_{02} \sin ^{2} \theta=0
$$

reduces (1.2) to a system of the special form. We still denote $\tilde{x}, \tilde{y}$ with $x, y$ for simple

$$
\begin{aligned}
\frac{d x}{d t}= & -y+x\left(\left(x^{2}+y^{2}\right)^{\lambda-1}\left(\tilde{A}_{11} x y\right)+\left(x^{2}+y^{2}\right)^{4(\lambda-1)}\right. \\
& \left.\times\left(\tilde{A}_{60} x^{6}+\tilde{A}_{51} x^{5} y+\tilde{A}_{42} x^{4} y^{2}+\tilde{A}_{33} x^{3} y^{3}+\tilde{A}_{24} x^{2} y^{4}+\tilde{A}_{15} x y^{5}+\tilde{A}_{06} y^{6}\right)\right), \\
\frac{d y}{d t}= & x+y\left(\left(x^{2}+y^{2}\right)^{\lambda-1}\left(\tilde{A}_{11} x y\right)+\left(x^{2}+y^{2}\right)^{4(\lambda-1)}\right. \\
& \left.\times\left(\tilde{A}_{60} x^{6}+\tilde{A}_{51} x^{5} y+\tilde{A}_{42} x^{4} y^{2}+\tilde{A}_{33} x^{3} y^{3}+\tilde{A}_{24} x^{2} y^{4}+\tilde{A}_{15} x y^{5}+\tilde{A}_{06} y^{6}\right)\right),
\end{aligned}
$$

whose coefficients are expressible in terms of the coefficients of (1.2). In particular, we have

$$
\tilde{A}_{11}=A_{11} \cos ^{2} \theta-4 A_{20} \cos \theta \sin \theta-A_{11} \sin ^{2} \theta .
$$

Using Theorem 3.2, we could get the center conditions of (3.1).

\section{Bifurcations of limit circle}

Now, we will discuss the bifurcations of limit circle at the origin of the system. It is obvious that the following theorem holds.

Theorem 4.1 The origin of system (3.1) is a fifth-order weak focus if and only if

$$
\begin{aligned}
& A_{44}=-\frac{5}{3}\left(7 A_{08}+A_{26}+A_{62}+7 A_{80}\right), \\
& A_{26}=-14 A_{08}+A_{62}+14 A_{80}, \\
& A_{62}=-7\left(A_{08}+3 A_{80}\right), \\
& A_{08}=A_{80}, \quad A_{11} A_{80} \neq 0 .
\end{aligned}
$$

Theorem 4.2 Suppose that $(0,0)$ is a fine focus of order five of system (3.1), the perturbed system (3.1) has at least five small amplitude limit circles.

Proof Under the conditions of Theorem 4.1, the Jacobian determinant of the function group $\left(u_{4}, u_{5}, u_{6}, u_{7}\right)$ with respect to variables $\left(A_{44}, A_{26}, A_{62}, A_{08}\right)$ has the following form:

$$
J=\frac{A_{11}^{6} \lambda^{10}}{4,194,304}
$$

because $A_{11} A_{80} \neq 0$. The conclusion of Theorem 4.2 follows according to the theory of the article [14]. 


\section{Competing interests}

The authors declare that they have no competing interests.

\section{Authors' contributions}

The authors have made the same contribution. All authors read and approved the final manuscript

\section{Acknowledgements}

This research is partially supported by the National Nature Science Foundation of China $(11201211,61273012)$ and Nature Science Foundation of Shandong Province (ZR2012AL04).

\section{Received: 17 October 2012 Accepted: 30 November 2012 Published: 28 December 2012}

\section{References}

1. Lin, Y, Li, J: Normal form and critical points values of the period of closed orbits for planar autonomous systems. Acta Math. Sin. 34, 490-501 (1991)

2. Conti, R: Uniform isochronous centers of polynomial systems in $R^{2}$. In: Differential Equations, Dynamical Systems, and Control Science. Lecture Notes in Pure and Appl. Math., vol. 152, pp. 21-31 (1994)

3. Chavarriga, J, Sabatini, M: A survey of isochronous centers. Qual. Theory Dyn. Syst. 1, 1-70 (1999)

4. Algaba, A, Reyes, M, Bravo, A: Geometry of the uniformly isochronous centers with polynomial commutators. Differ. Equ. Dyn. Syst. 10, 257-275 (2002)

5. Algaba, A, Reyes, M: Computing center conditions for vector fields with constant angular speed. J. Comput. Appl. Math. 154, 143-159 (2003)

6. Conti, R: Centers of planar polynomial systems, a review. Matematiche 53, 207-240 (1998)

7. Libre, J, Valls, C: Classification of the centers, their cyclicity and isochronicity for a class of polynomial differential systems generalizing the linear systems with cubic homogeneous nonlinearities. J. Differ. Equ. 246, 2192-2204 (2009)

8. Libre, J, Valls, C: Classification of the centers and isochronous centers for a class of quartic-like systems. Nonlinear Anal. 71, 3119-3128 (2009)

9. Libre, J, Valls, C: Classification of the centers, their cyclicity and isochronicity for the generalized quadratic polynomial differential systems. J. Math. Anal. Appl. 357, 427-437 (2009)

10. Libre, J, Valls, C: Classification of the centers, of their cyclicity and isochronicity for two classes of generalized quintic polynomial differential systems. Nonlinear Differ. Equ. Appl. 16, 657-679 (2009)

11. Libre, J, Valls, C: Centers and isochronous centers for two classes of generalized seventh and ninth systems. J. Dyn. Differ. Equ. 22, 657-675 (2010)

12. Chen, $X$, Huang, W: Linearizability conditions of a time-reversible quartic-like system. J. Math. Anal. Appl. (2011). doi:10.1016/j.jmaa.2011.05.018

13. Liu, Y, Li, J: Theory of values of singular point in complex autonomous differential system. Sci. China Ser. A 3, 245-255 (1989)

14. Liu, Y, Li, J: Theory of values of singular point in complex autonomous differential system. Sci. China Ser. A 3, 245-255 (1989)

doi:10.1186/1687-1847-2012-231

Cite this article as: Ren et al.: Computing center conditions for non-analytic ninth degree vector fields with constant angular speed. Advances in Difference Equations 2012 2012:231.

\section{Submit your manuscript to a SpringerOpen ${ }^{\ominus}$ journal and benefit from:}

- Convenient online submission

- Rigorous peer review

- Immediate publication on acceptance

- Open access: articles freely available online

- High visibility within the field

- Retaining the copyright to your article 